\title{
Clinical features and pathogenesis of membranoproliferative glomerulonephritis: a nationwide analysis of the Japan renal biopsy registry from 2007 to 2015
}

\author{
Naoki Nakagawa' ${ }^{1}$ (D) Naoyuki Hasebe ${ }^{1} \cdot$ Motoshi Hattori $^{2} \cdot$ Michio Nagata $^{3} \cdot$ Hitoshi Yokoyama $^{4} \cdot$ Hiroshi Sato $^{5} \cdot$ \\ Hitoshi Sugiyama $^{6} \cdot$ Akira Shimizu $^{7} \cdot$ Yoshitaka Isaka $^{8} \cdot$ Shoichi Maruyama $^{9} \cdot$ Ichiei Narita $^{10}$
}

Received: 24 September 2017 / Accepted: 19 November 2017 / Published online: 6 December 2017

(c) The Author(s) 2017. This article is an open access publication

\begin{abstract}
Background The incidence and age distribution of membranoproliferative glomerulonephritis (MPGN) vary throughout the world by race and ethnicity. We sought to evaluate the clinical features, pathogenesis, and age distribution of MPGN among a large nationwide data from the Japan Renal Biopsy Registry (J-RBR).

Methods A cross-sectional survey of 593 patients with MPGN (types I and III) registered in the J-RBR between 2007 and 2015 was conducted. Clinical parameters, and laboratory findings at diagnosis were compared between children ( $<20$ years), adults (20-64 years), and elderly patients ( $\geq 65$ years).

Results The median age of the patients was 59.0 years and mean proteinuria was $3.7 \mathrm{~g} /$ day. The rate of nephrotic syndrome was significantly higher in adults (40.4\%) and elderly patients (54.0\%) than in children (14.9\%), whereas the rate of chronic glomerulonephritis was significantly higher in children (66.2\%) than in adults (34.4\%) and elderly patients (31.2\%). According to the CGA risk classification, high-risk (red zone) cases accounted for $3.4 \%$ of children, $52.5 \%$ of adults and $84.1 \%$ of elderly patients with MPGN. As for pathogenesis, primary MPGN was most frequent (56.0\%). Lupus nephritis was the most common disease among adult patients with secondary MPGN, whereas infectious disease was more common in elderly patients. Multiple regression analysis revealed that high systolic blood pressure and high proteinuria were independent factors associated with decreased estimated glomerular filtration rate (eGFR) in adults and elderly patients with MPGN.

Conclusions In Japan, adults and elderly patients with MPGN had a lower eGFR and severer proteinuria than children.
\end{abstract}

Keywords Membranoproliferative glomerulonephritis $\cdot$ Renal pathology $\cdot$ Registry $\cdot$ Age distribution

Naoki Nakagawa

naka-nao@asahikawa-med.ac.jp

1 Division of Cardiology and Nephrology, Department of Internal Medicine, Asahikawa Medical University, Asahikawa, Japan

2 Department of Pediatric Nephrology, Tokyo Women's Medical University, Shinjuku-ku, Tokyo, Japan

3 Department of Kidney and Vascular Pathology, Faculty of Medicine, University of Tsukuba, Tsukuba, Japan

4 Division of Nephrology, Kanazawa Medical University School of Medicine, Uchinada, Japan

5 Clinical Pharmacology and Therapeutics, Tohoku University, Graduate School of Pharmaceutical Sciences, Sendai, Japan
6 Department of Medicine and Clinical Science, Okayama University Graduate School of Medicine, Dentistry, and Pharmaceutical Sciences, Okayama, Japan

7 Department of Analytic Human Pathology, Nippon Medical School, Bunkyo-ku, Tokyo 113-8602, Japan

8 Department of Nephrology, Osaka University Graduate School of Medicine, Suita, Osaka, Japan

9 Department of Nephrology, Nagoya University Graduate School of Medicine, Nagoya, Japan

10 Division of Clinical Nephrology and Rheumatology, Niigata University Graduate School of Medical and Dental Sciences, Niigata, Japan 


\section{Introduction}

Membranoproliferative glomerulonephritis (MPGN), also termed as mesangiocapillary glomerulonephritis, is a morphological pattern of injury characterized by mesangial hypercellularity, endocapillary proliferation, capillary wall remodeling, double contour formation, and duplication of basement membranes on light microscopy [1,2]. In the clinical setting, MPGN is one of the most common causes of nephrotic syndrome in both children and adults, and accounts for approximately $2-10 \%$ of all cases of biopsy-confirmed glomerulonephritis [3-6]. Traditionally, MPGN has been classified based on the findings of electron microscopy as primary MPGN type I (MPGN I), MPGN II, MPGN III, or secondary MPGN [1, 2]. MPGN I, the most common form, is characterized by subendothelial deposits, and MPGN III has both subepithelial and subendothelial deposits, whereas MPGN type II is characterized by dense deposits in the glomerular basement membrane (dense deposit disease [DDD]) [1,2]. Together with DDD, this group of C3-positive immunoglobulinnegative glomerular diseases has been labeled as $\mathrm{C} 3$ glomerulopathies [7, 8]. Furthermore, the MPGN has the strongest association with secondary causes, including viruses, autoimmune diseases, and paraproteins [1,9]. However, the clinical features and pathogenesis of MPGN in an adequate sample of patients at different ages have not been studied in detail. We, therefore, surveyed the clinical features and pathogenesis of MPGN types I and III based on data between July 2007 and June 2015 from the Japan Renal Biopsy Registry (J-RBR), which is a nationwide, web-based, prospective registry of renal biopsies.

\section{Methods}

\section{Registry description}

The J-RBR was established by the Committee for the Standardization of Renal Pathological Diagnosis and the Working Group for the Renal Biopsy Database of the Japanese Society of Nephrology in 2007 [10]. Patient data were registered on the J-RBR website using the Internet Data and Information Center for Medical Research (INDICE) system of the University Hospital Medical Information System (UMIN). The J-RBR is registered under the Clinical Trial Registry of UMIN (Registration Number, UMIN000000618), and the Ethics Review Board of the Japanese Society of Nephrology approved the present study in accordance with the Declaration of Helsinki. Written informed consent was obtained from all the patients at the time they were registered to participate in the study. Among the 26,535 patients with biopsy-proven disease who were registered in this system between July 2007 and June 2015, we selected 593 (2.2\%) patients with MPGN who were registered as having a histopathology of MPGN types I and III. The patients were classified as children, adults, and elderly according to age $<20$ years, $20-64$ years, and $\geq 65$ years, respectively.

\section{Clinical diagnoses and parameters}

According to the modified classification of World Health Organization, primary glomerular diseases were mainly clinically diagnosed as chronic nephritic syndrome, acute nephritic syndrome, recurrent or persistent hematuria, rapidly progressive nephritic syndrome, and nephrotic syndrome [10]. Secondary glomerular diseases were categorized as renal disorders with collagen disease or vasculitis, renal disease with metabolic syndrome, hypertensive nephropathy, acute kidney injury, drug-induced nephropathy, thrombotic microangiopathy, and others. The J-RBR requires classification based on pathogenesis and histopathology. All the extracted patients were registered as having a histopathology of MPGN type I and III. Patients with DDD were excluded from this study.

\section{Evaluation of other clinical findings}

The registered basic information (age, sex, height, and weight) as well as urinary findings (urinalysis, daily proteinuria), blood findings (serum creatinine, total protein, serum albumin, total cholesterol), blood pressure (BP) and the presence of concomitant hypertension and diabetes were assessed in the present study. Estimated glomerular filtration rate (eGFR) was calculated using the modified equations for Japanese children [11] and for Japanese adults [12]. Information about prescribed anti-hypertensive agents, the presence of diabetes mellitus, and $\mathrm{HbA} 1 \mathrm{c}$ were arbitrarily registered. We analyzed the frequency of high-risk (red zone) cases of primary MPGN according to patient age, based on the CGA risk classification for CKD (the heat map method), as described previously [13].

\section{Statistical analysis}

Continuous variables, except age, are presented as mean \pm standard deviation. Age is expressed as median and interquartile range. Clinical parameters were compared among the three patient age groups using a single-factor analysis of variance for normally distributed continuous variables or the Kruskal-Wallis test for non-normally distributed continuous variables. The normality of the variances for each continuous variable was analyzed using Levene 
test. Differences in proportions were evaluated using the Chi square independent test or Fisher's exact test, depending on the number of categories. Independent factors affecting renal function at diagnosis were evaluated using stepwise multiple regression analyses. Quantitative variables such as body mass index calculated by height and weight, proteinuria, systolic BP, serum albumin, and serum total cholesterol were selected as independent variables in the analyses. Age, sex, and the value of serum creatinine were excluded, because these variables were used in the equation of eGFR. All data were statistically analyzed using IBM SPSS Advanced Statistical Version 19.0 (SPSS, Chicago, IL, USA), and $p<0.05$ was considered to indicate a significant difference.

\section{Results}

\section{General data of patients}

Patient demographics of MPGN in Japan are shown in Table 1 and Fig. 1. Among the 593 patients with MPGN, $319(53.8 \%)$ were male and $274(46.2 \%)$ were female. The median age of the overall patients was 59.0 years ( 61.0 years for men and 56.0 years for women). The number of registered patients increased with age and peaked in the seventh decade for the overall patients and male patients, whereas it peaked in the eighth decade for female patients (Fig. 1a).

Table 1 Patient demographics of membranoproliferative glomerulonephritis in Japan (J-RBR 2007-2015)

\begin{tabular}{llllrr}
\hline & $N$ & Min & Max & Mean & SD \\
\hline Age (years old) & 593 & 3 & 89 & 52.7 & 22.4 \\
Male & 319 & 3 & 89 & 54.1 & 21.2 \\
Female & 274 & 4 & 88 & 51.0 & 23.6 \\
Height (cm) & 582 & 94.5 & 186.1 & 157.8 & 12.7 \\
Weight (kg) & 581 & 15.2 & 107.6 & 57.0 & 13.8 \\
Body mass index (BMI) & 581 & 10.4 & 37.6 & 22.6 & 3.8 \\
Proteinuria (g/day) & 429 & 0.0 & 19.4 & 3.7 & 3.2 \\
Urinary protein/creatinine ratio & 444 & 0.0 & 32.0 & 4.8 & 4.2 \\
Serum creatinine (mg/dl) & 593 & 0.2 & 9.7 & 1.3 & 1.0 \\
eGFR (mL/min/1.73 m $\left.{ }^{2}\right)$ & 588 & 6.4 & 161.3 & 57.8 & 32.1 \\
Serum total protein (g/dl) & 592 & 3.3 & 9.9 & 5.8 & 1.0 \\
Serum albumin (g/dl) & 592 & 1.1 & 5.0 & 2.9 & 0.8 \\
Serum total cholesterol (mg/dl) & 575 & 78.0 & 558.0 & 225.4 & 72.8 \\
Systolic blood pressure (mmHg) & 507 & 87.0 & 227.0 & 138.1 & 21.5 \\
Diastolic blood pressure (mmHg) & 507 & 38.0 & 114.0 & 77.4 & 13.3 \\
Mean blood pressure (mmHg) & 507 & 57.0 & 146.3 & 97.6 & 14.2 \\
HbA1c (\%) & 366 & 3.8 & 14.2 & 5.7 & 0.8 \\
\hline
\end{tabular}

$J-R B R$ Japan Renal Biopsy Registry, eGFR estimated glomerular filtration rate, $H b A l c$ hemoglobin Alc
The urinary findings of the patients are shown in Table 2. In dipstick tests, 36 cases $(6.1 \%)$ were classified as (-) or $( \pm)$, and 377 cases $(63.6 \%)$ were classified as $\geq 3+$. Similarly, 28 cases $(6.5 \%)$ and 18 cases $(4.1 \%)$ involved patients who exhibited daily proteinuria values of $<0.3 \mathrm{~g} /$ day or urinary protein/creatinine ratios (UPCR) of $<0.3 \mathrm{~g} / \mathrm{gCr}$ (spot urine tests). In addition, 385 cases (89.8\%) and 412 cases $(92.8 \%)$ involved patients who demonstrated daily proteinuria values of $\geq 0.5 \mathrm{~g} /$ day and UPCR of $\geq 0.5 \mathrm{~g} / \mathrm{gCr}$, respectively.

In this study, the mean daily proteinuria was $3.7 \mathrm{~g}$, mean UPCR was $4.8 \mathrm{~g} / \mathrm{gCr}$, mean serum albumin level was $2.9 \mathrm{~g} /$ $\mathrm{dL}$, and mean eGFR was $57.8 \mathrm{~mL} / \mathrm{min} / 1.73 \mathrm{~m}^{2}$ (Table 1). When we judged the nephrotic state of the patients based on the new criteria for nephrotic syndrome used in Japan [14]; i.e., daily proteinuria (or a UPCR) of more than $3.5 \mathrm{~g}$ (or g/ $\mathrm{gCr}$ ) and serum albumin levels less than $3.0 \mathrm{~g} / \mathrm{dL}$ or serum total protein levels less than $6.0 \mathrm{~g} / \mathrm{dL}, 297$ (253 patients with nephrotic syndrome and 44 patients with nephrotic syndrome with collagen disease/vasculitis)(50.1\%) of the 593 patients with MPGN were considered to be nephrotic (Fig. 1c; Table 3). The frequency of nephrotic state increased with age, from $10 \%$ in the first decade to $70 \%$ in the ninth decade (Fig. 1c).

With regard to hematuria, 123 cases $(20.8 \%)$ were found to be $(-)$ or $( \pm)$ for occult blood in dipstick tests, and 386 cases $(65.1 \%)$ were classified as $\geq 2+$. Similarly, 156 cases (26.3\%) were considered to be red blood cell (RBC)-negative or to have $<5 \mathrm{RBC} /$ high-powered field (hpf) in their urinary sediments, and 169 cases $(28.5 \%)$ and 170 cases (28.7\%) were considered to have $<10-30 / \mathrm{hpf}$ and many/hpf, respectively (Table 2).

\section{Clinical features and pathogenesis of MPGN}

The main clinical diagnoses of the overall patients with MPGN at the time of renal biopsy were nephrotic syndrome in 253 patients (42.7\%), chronic nephritic syndrome in 220 patients (37.1\%), nephrotic syndrome with collagen disease/ vasculitis in 44 patients (7.4\%), and chronic nephritic syndrome with collagen disease/vasculitis in 34 patients $(5.7 \%)$ (Table 3).

In the present analysis, $332(56.0 \%)$ of the 593 patients were registered as "primary" MPGN, whereas 261 patients (44.0\%) were registered as "secondary" MPGN (Table 3), including $74(12.5 \%)$ patients with lupus nephritis, 72 patients $(12.1 \%)$ with infectious disease (hepatitis B virus: 5, hepatitis $\mathrm{C}$ virus: 38 , and shunt infection: 3 ), and 29 patients (4.9\%) with IgA nephropathy (Fig. 1b; Table 3). In the remarks columns of the database, there were 32 patients (5.2\%) with cryoglobulinemia, 5 patients with light-chain deposition disease, 3 patients with heavy-chain deposition disease, and 2 patients with $\mathrm{C} 3$ glomerulopathies. As for the 


\section{a}

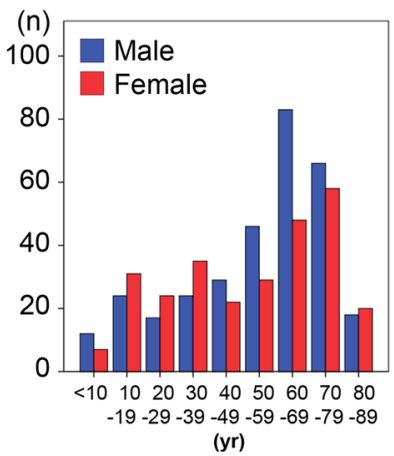

C

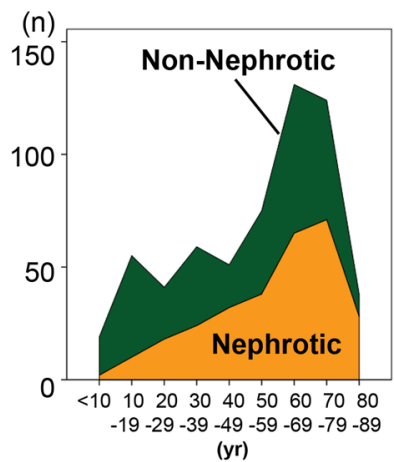

f

aged under 20 years

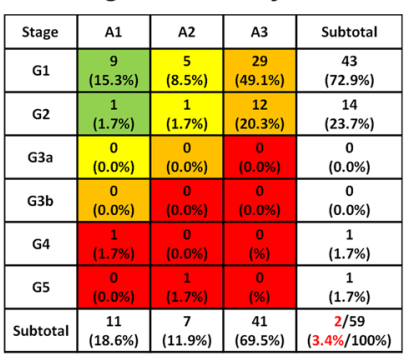

\section{b}

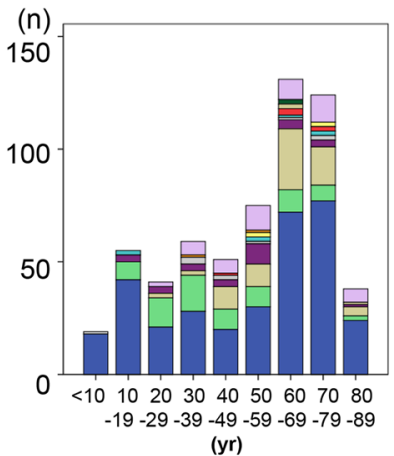

d

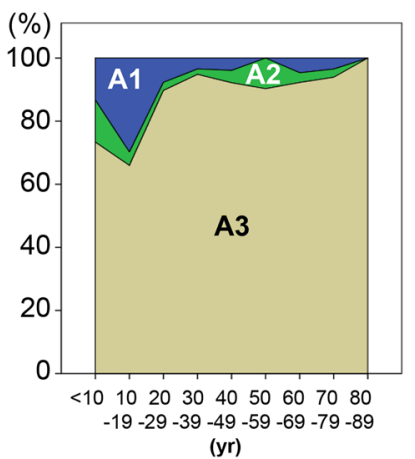

g

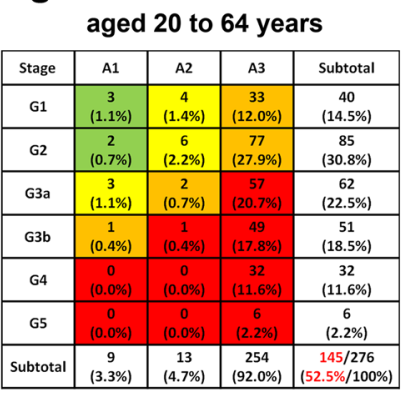

Primary MPGN

Lupus nephritis

Infection-related nephropathy

IgA nephropathy

Thrombotic microangiopathy

Purpura nephritis

Diabetic nephropathy

Amyroidosis

Hypertensive nephrosclerosis

MPO-ANCA-positive nephritis

Transplanted kidney

Others

\section{e}

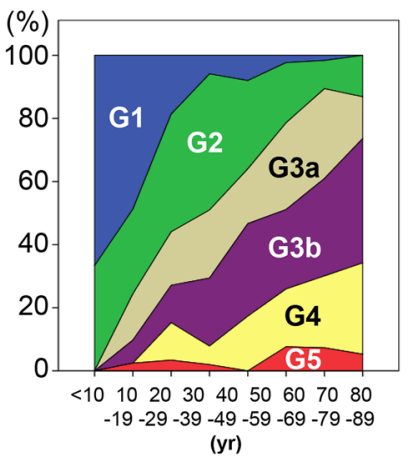

h

\begin{tabular}{|c|c|c|c|c|}
\multicolumn{5}{|c|}{ aged over 65 years } \\
\hline Stage & A1 & A2 & A3 & Subtotal \\
\hline \multirow{2}{*}{ G1 } & 0 & 0 & 4 & 4 \\
& $(0.0 \%)$ & $(0.0 \%)$ & $(1.8 \%)$ & $(1.8 \%)$ \\
\hline \multirow{2}{*}{ G2 } & $\begin{array}{c}1 \\
(0.4 \%)\end{array}$ & $\begin{array}{c}0 \\
(0.0 \%)\end{array}$ & $\begin{array}{c}25 \\
(11.1 \%)\end{array}$ & $\begin{array}{c}26 \\
(11.5 \%)\end{array}$ \\
\hline \multirow{2}{*}{ G3a } & $\begin{array}{c}3 \\
(1.3 \%)\end{array}$ & $\begin{array}{c}1 \\
(0.4 \%)\end{array}$ & $\begin{array}{c}47 \\
(20.8 \%)\end{array}$ & $\begin{array}{c}51 \\
(22.6 \%)\end{array}$ \\
\hline \multirow{2}{*}{ G3b } & 2 & 2 & 72 & 76 \\
$(0.9 \%)$ & $(0.9 \%)$ & $(31.9 \%)$ & $(33.6 \%)$ \\
\hline \multirow{2}{*}{ G4 } & 2 & 2 & 48 & 52 \\
& $(0.9 \%)$ & $(0.9 \%)$ & $(21.2 \%)$ & $(23.0 \%)$ \\
\hline \multirow{2}{*}{ G5 } & 0 & 0 & 17 & 17 \\
& $(0.0 \%)$ & $(0.0 \%)$ & $(7.5 \%)$ & $(7.5 \%)$ \\
\hline \multirow{2}{*}{ Subtotal } & 8 & 5 & 213 & $190 / 226$ \\
$(3.5 \%)$ & $(2.2 \%)$ & $(94.2 \%)$ & $(84.1 \% 100 \%)$ \\
\hline
\end{tabular}

Fig. 1 Age distribution and clinical features of membranoproliferative glomerulonephritis (MPGN) in the J-RBR. a The number of cases peaked in the seventh decade for the overall patients and male patients, whereas they peaked in the eighth decade for female patients. b The number of pathogenesis of MPGN. The number of cases with primary MPGN, lupus nephritis, and infectious disease peaked in the eighth, fourth, and seventh decade, respectively. Lupus nephritis was the most common disease among patients with secondary MPGN in their third and fourth decade, whereas infectious disease was the most common in their fifth decade and beyond. c The number of nephrotic syndrome in patients with MPGN, and

age distribution of the MPGN patients, more than $80 \%$ of the patients in their first and second decade were registered as primary MPGN, whereas around $60 \%$ of the patients in their fourth and fifth decade were registered as secondary MPGN (Fig. 1b). Lupus nephritis was the most common disease among patients with secondary MPGN in their third and fourth decade, whereas infectious disease was more common in the fifth decade and beyond (Fig. 1b). age distribution of the population. The frequency of nephrotic state increased with age. $\mathbf{d}-\mathbf{e}$ chronic kidney disease (CKD) A stages (d) and $\mathrm{G}$ stages (e) of MPGN. More than $90 \%$ of patients aged 20 years and above had severe proteinuria (A3) (d). The number of patients with advanced CKD (stage G3a, G3b, G4, or G5) increased with age, and more than $70 \%$ of them were 60 years old or older (e). f-h The CGA risk classification of MPGN. According to the CGA risk classification, high-risk (red zone) cases accounted for $3.4 \%$ of patients aged under 20 years (f), $52.5 \%$ of patients aged 20 to 64 years (g) and $84.1 \%$ of those aged over 65 years $(\mathbf{h})$

Around $70 \%$ of patients aged less than 20 years and more than $90 \%$ of patients aged more than 20 years had severe proteinuria (A3)(Fig. 1d). The number of patients with advanced chronic kidney disease (CKD)(stage G3a to G5) increased with age, and more than $70 \%$ of the patients were 60 years old or older (Fig. 1e). According to the CGA risk classification, high-risk (red zone) cases accounted for $3.4 \%$ of children, $52.5 \%$ of adults and $84.1 \%$ of elderly 
Table 2 Urinalysis results of all cases of membranoproliferative glomerulonephritis in Japan (J-RBR 2007-2015)

\begin{tabular}{|c|c|c|c|c|c|}
\hline \multicolumn{4}{|c|}{ Urinary protein (dipstick test) } & Cases & $\%$ \\
\hline \multicolumn{4}{|l|}{$(-)$} & 16 & 2.7 \\
\hline \multicolumn{4}{|l|}{$( \pm)$} & 20 & 3.4 \\
\hline \multicolumn{4}{|l|}{$1+$} & 48 & 8.1 \\
\hline \multicolumn{4}{|l|}{$2+$} & 132 & 22.3 \\
\hline \multicolumn{4}{|l|}{$3+$} & 258 & 43.5 \\
\hline \multicolumn{4}{|l|}{$4+$} & 119 & 20.1 \\
\hline \multicolumn{4}{|l|}{ Total } & 593 & 100.0 \\
\hline \multicolumn{6}{|c|}{ Daily proteinuria levels and urinary protein levels according to spot urine tests } \\
\hline g/day & Cases & $\%$ & $\mathrm{~g} / \mathrm{g} \mathrm{Cr}$ & Cases & $\%$ \\
\hline$<0.3$ & 28 & 6.5 & $<0.3$ & 18 & 4.1 \\
\hline \multirow{2}{*}{$\begin{array}{l}0.3-0.49 \\
0.50-0.99\end{array}$} & 16 & 3.7 & $0.3-0.49$ & 14 & 3.2 \\
\hline & 44 & 10.3 & $0.50-0.99$ & 30 & 6.8 \\
\hline $1.0-3.49$ & 149 & 34.7 & $1.0-3.49$ & 159 & 35.8 \\
\hline \multirow{2}{*}{$\begin{array}{l}3.5+ \\
\text { Total }\end{array}$} & 192 & 44.8 & $3.5+$ & 223 & 50.2 \\
\hline & 429 & 100.0 & Total & 444 & 100.0 \\
\hline \multicolumn{6}{|c|}{ Hematuria (occult blood grade and red blood cell grade of urinary sediment) } \\
\hline OB & Cases & $\%$ & /hpf & Cases & $\%$ \\
\hline$(-)$ & 58 & 9.8 & $(-)$ & 26 & 4.4 \\
\hline$( \pm)$ & 65 & 11.0 & $<5$ & 130 & 21.9 \\
\hline $1+$ & 84 & 14.2 & $5-10$ & 98 & 16.5 \\
\hline $2+$ & 169 & 28.5 & $<10-30$ & 169 & 28.5 \\
\hline \multirow{2}{*}{$\begin{array}{l}3+ \\
\text { Total }\end{array}$} & 217 & 36.6 & Many & 170 & 28.7 \\
\hline & 593 & 100 & Total & 593 & 100.0 \\
\hline
\end{tabular}

$J$-RBR Japan Renal Biopsy Registry, $O B$ occult blood levels according to the dipstick test, $h p f$ high powered field

patients with MPGN (Fig. 1f-h), suggesting that compared to children, adults and elderly patients with MPGN have more severe renal involvement according to advanced age.

Table 4 compares the clinical features between children, adults, and elderly patients with MPGN. The rate of nephrotic syndrome was significantly higher in adults (40.4\%) and elderly patients $(54.0 \%)$ than in children (14.9\%), whereas the rate of chronic glomerulonephritis was significantly higher in children $(66.2 \%)$ than in adults $(34.4 \%)$ and elderly patients $(31.2 \%)$. The number of patients with hypertension, large amount of proteinuria, reduced renal function, and hypoalbuminemia distinctly differed among the age groups, and disease severity was worse in elderly patients with MPGN. In contrast, the ratio of overt hematuria ( $>30 / \mathrm{hpf}$ ) (40.5 vs. 24.5 vs. $29.5 \%$ ) was worse in children with MPGN (Table 4), suggesting that children are more likely to have hematuria at onset and less likely to have renal insufficiency and hypertension, as previously reported [15].

\section{Clinical features of primary MPGN}

Next, we focused on only primary MPGN. Of the 26,535 total renal biopsies, 332 patients were registered as primary MPGN (1.3\%). Among these, 185 patients (55.7\%) were male and 147 (44.3\%) were female. The median age of the patients was 61.0 years. The number of patients increased with age and peaked in the eighth decade for the overall patients and female patients, whereas it peaked in the seventh decade for male patients (Fig. 2a). The mean proteinuria was $3.8 \mathrm{~g} /$ day, mean serum albumin was $3.0 \mathrm{~g} / \mathrm{dL}$, and mean eGFR was $59.0 \mathrm{~mL} / \mathrm{min} / 1.73 \mathrm{~m}^{2}$ (Table 5).

The main clinical diagnoses of the patients with primary MPGN were nephrotic syndrome in 163 patients (49.1\%) and chronic nephritic syndrome in 148 patients (44.6\%). The diagnosis of nephrotic syndrome was found to increase with age, whereas that of chronic nephritic syndrome decreased with age in patients with primary MPGN. Around 70\% of patients aged less than 20 years and more than $90 \%$ of patients aged more than 20 years had severe proteinuria (A3) (Fig. 2b). The number of patients with advanced CKD (stage G3a to G5) increased with age, and more than $80 \%$ of 
Table 3 Clinical diagnoses and pathogenesis of membranoproliferative glomerulonephritis in Japan (J-RBR 2007-2015)

\begin{tabular}{|c|c|c|}
\hline & Cases & $\%$ \\
\hline \multicolumn{3}{|l|}{ Clinical diagnoses } \\
\hline Nephrotic syndrome & 253 & 42.7 \\
\hline Chronic nephritic syndrome & 220 & 37.1 \\
\hline Nephrotic syndrome + collagen disease/vasculitis & 44 & 7.4 \\
\hline Chronic nephritic syndrome + collagen disease/vasculitis & 34 & 5.7 \\
\hline Rapidly progressive nephritic syndrome & 19 & 3.2 \\
\hline Acute nephritic syndrome & 12 & 2.0 \\
\hline Recurrent hematuria & 5 & 0.8 \\
\hline HUS/TTP & 2 & 0.3 \\
\hline Acute kidney injury & 2 & 0.3 \\
\hline Acute nephritic syndrome + collagen disease/vasculitis & 1 & 0.2 \\
\hline Rapidly progressive nephritic syndrome + collagen disease/vasculitis & 1 & 0.2 \\
\hline Total & 593 & 100 \\
\hline \multicolumn{3}{|l|}{ Pathogenesis } \\
\hline Primary (idiopathic) & 332 & 56.0 \\
\hline Lupus nephritis & 74 & 12.5 \\
\hline Infection-related nephropathy & 72 & 12.1 \\
\hline IgA nephropathy & 29 & 4.9 \\
\hline Thrombotic microangiopathy & 8 & 1.3 \\
\hline Purpura nephritis & 7 & 1.2 \\
\hline Diabetic nephropathy & 6 & 1.0 \\
\hline Amyloidosis & 4 & 0.7 \\
\hline Hypertensive nephrosclerosis & 3 & 0.5 \\
\hline MPO-ANCA-positive nephritis & 2 & 0.3 \\
\hline Transplanted kidney & 2 & 0.3 \\
\hline Others & 54 & 9.1 \\
\hline Total & 593 & 100 \\
\hline
\end{tabular}

$J$-RBR Japan Renal Biopsy Registry, HUS/TTP hemolytic uremic syndrome/thrombotic thrombocytopenic purpura, $M P O-A N C A$ myeloperoxidaseantineutrophil cytoplasmic antibody these patients were 60 years old or older (Fig. 2c). According to the CGA risk classification, high-risk (red zone) cases accounted for $4.0 \%$ of children, $53.9 \%$ of adults and $84.7 \%$ of elderly patients with primary MPGN (Fig. 2d-f) as similar to overall MPGN.

The ratios of nephrotic syndrome (16.7 vs. 48.8 vs. $62.9 \%$ ) were significantly higher, whereas those of chronic nephritic syndrome (76.7 vs. 46.5 vs. $29.4 \%$ ) were significantly lower in elderly patients than in adults and children (Table 6). The disease severity was worse in elderly patients with primary MPGN as similar to overall MPGN.

\section{Impact of clinical features on renal function}

As for hypertension, BP and/or the intake of anti-hypertensive drugs were registered in 520 patients with MPGN. Hypertension, as judged by a systolic BP of more than
$140 \mathrm{mmHg}$, a diastolic BP of more than $90 \mathrm{mmHg}$, or drug intake, was observed in 374 (71.9\%) patients with MPGN.

The impact of clinical factors on renal function at the time of biopsy was evaluated using multiple regression analysis. The model included important risk factors for progression of renal function as imperative independent variables. Higher systolic BP and serum total cholesterol, and increased proteinuria at diagnosis were significant factors for a decline in renal function in adult and elderly patients with overall MPGN $\left(R^{2}=0.104 ; F=13.021\right.$, $p<0.001$; Table 7a) and with primary MPGN $\left(R^{2}=0.157\right.$; $F=10.791, p<0.001$; Table 7b).

\section{Discussion}

This is the first study to characterize the clinical features using the CGA risk classification and pathogenesis of MPGN in a large nationwide registry of renal biopsies. 
Table 4 Comparison of clinical features and pathogenesis according to age in patients with membranoproliferative glomerulonephritis

\begin{tabular}{|c|c|c|c|c|}
\hline & Child & Adult & Elderly & $P$ \\
\hline$N$ & 74 & 282 & 237 & \\
\hline Age (years old) & $11.9 \pm 4.1$ & $46.0 \pm 13.3$ & $73.4 \pm 5.7$ & $<0.001^{*}$ \\
\hline Male/female & $36 / 38$ & $158 / 124$ & $125 / 112$ & 0.483 \\
\hline Body mass index (BMI) & $18.8 \pm 3.1$ & $23.2 \pm 3.6$ & $23.1 \pm 3.6$ & $<0.001^{*}$ \\
\hline Systolic blood pressure (mmHg) & $113.0 \pm 15.0$ & $135.6 \pm 18.7$ & $147.8 \pm 19.8$ & $<0.001^{*}$ \\
\hline Diastolic blood pressure $(\mathrm{mmHg})$ & $64.7 \pm 10.8$ & $80.0 \pm 12.9$ & $77.6 \pm 12.4$ & $<0.001^{*}$ \\
\hline Patients with hypertension at diagnosis & $17.3 \%$ & $60.5 \%$ & $86.4 \%$ & $<0.001 *$ \\
\hline Sediment RBC (> 30/hpf, \%) & $40.5 \%$ & $24.5 \%$ & $29.5 \%$ & $0.027 *$ \\
\hline Proteinuria (g/day) & $1.7 \pm 2.1$ & $3.8 \pm 3.0$ & $3.9 \pm 3.5$ & $<0.001^{*}$ \\
\hline Urinary protein/creatinine ratio & $2.6 \pm 3.9$ & $4.5 \pm 3.6$ & $5.7 \pm 4.8$ & $<0.001 *$ \\
\hline Serum creatinine $(\mathrm{mg} / \mathrm{dl})$ & $0.71 \pm 1.11$ & $1.22 \pm 0.75$ & $1.56 \pm 1.08$ & $<0.001^{*}$ \\
\hline eGFR (mL/min/1.73 m²) & $106.2 \pm 28.9$ & $60.4 \pm 28.4$ & $40.3 \pm 18.7$ & $<0.001^{*}$ \\
\hline Serum albumin $(\mathrm{g} / \mathrm{dl})$ & $3.4 \pm 0.9$ & $2.9 \pm 0.8$ & $2.7 \pm 0.7$ & $<0.001^{*}$ \\
\hline Serum total cholesterol $(\mathrm{mg} / \mathrm{dl})$ & $216.9 \pm 74.4$ & $229.5 \pm 77.5$ & $223.2 \pm 66.2$ & 0.354 \\
\hline \multicolumn{5}{|l|}{ Clinical diagnosis } \\
\hline Nephrotic syndrome & $11(14.9 \%)$ & $114(40.4 \%)$ & $128(54.0 \%)$ & $<0.001^{*}$ \\
\hline Chronic nephritic syndrome & $49(66.2 \%)$ & $97(34.4 \%)$ & $74(31.2 \%)$ & $<0.001^{*}$ \\
\hline Nephrotic syndrome + collagen disease/vasculitis & $3(4.1 \%)$ & $30(10.6 \%)$ & $11(4.6 \%)$ & $0.017 *$ \\
\hline Chronic nephritic syndrome + collagen disease/vasculitis & $6(8.1 \%)$ & $25(8.9 \%)$ & $3(1.3 \%)$ & $0.001 *$ \\
\hline RPGN & $1(1.4 \%)$ & $7(2.5 \%)$ & $11(4.6 \%)$ & 0.238 \\
\hline Acute nephritic syndrome & $1(1.4 \%)$ & $5(1.8 \%)$ & $6(2.5 \%)$ & 0.753 \\
\hline Recurrent hematuria & $2(2.7 \%)$ & $2(0.7 \%)$ & $1(0.4 \%)$ & 0.163 \\
\hline HUS/TTP & $1(1.4 \%)$ & $0(0.0 \%)$ & $1(0.4 \%)$ & 0.195 \\
\hline Acute kidney injury & $0(0.0 \%)$ & $1(0.4 \%)$ & $1(0.4 \%)$ & 0.859 \\
\hline Acute nephritic syndrome + collagen disease/vasculitis & $0(0.0 \%)$ & $0(0.0 \%)$ & $1(0.4 \%)$ & 0.471 \\
\hline RPGN + collagen disease/vasculitis & $1(1.4 \%)$ & $7(2.5 \%)$ & $11(4.6 \%)$ & 0.238 \\
\hline \multicolumn{5}{|l|}{ Pathological diagnosis } \\
\hline Primary (idiopathic) & $60(81.1 \%)$ & $129(45.7 \%)$ & $143(60.3 \%)$ & $<0.001 *$ \\
\hline Lupus nephritis & $8(10.8 \%)$ & $52(18.4 \%)$ & $14(5.9 \%)$ & $<0.001 *$ \\
\hline Infection-related nephropathy & $0(0.0 \%)$ & $37(13.1 \%)$ & $35(14.8 \%)$ & $0.002 *$ \\
\hline IgA nephropathy & $3(4.1 \%)$ & $20(7.1 \%)$ & $6(2.5 \%)$ & 0.053 \\
\hline Thrombotic microangiopathy & $1(0.0 \%)$ & $6(1.8 \%)$ & $3(1.3 \%)$ & 0.495 \\
\hline Purpura nephritis & $2(2.7 \%)$ & $3(1.1 \%)$ & $2(0.8 \%)$ & 0.420 \\
\hline Diabetic nephropathy & $0(0.0 \%)$ & $1(0.4 \%)$ & $5(2.1 \%)$ & 0.090 \\
\hline Amyloidosis & $0(0.0 \%)$ & $2(0.7 \%)$ & $2(0.8 \%)$ & 0.737 \\
\hline Hypertensive nephrosclerosis & $0(0.0 \%)$ & $0(0.0 \%)$ & $3(1.3 \%)$ & 0.104 \\
\hline MPO-ANCA-positive nephritis & $0(0.0 \%)$ & $1(0.4 \%)$ & $1(0.4 \%)$ & 0.859 \\
\hline Transplanted kidney & $0(0.0 \%)$ & $2(0.7 \%)$ & $0(0.0 \%)$ & 0.331 \\
\hline Others & $0(0.0 \%)$ & $29(10.3 \%)$ & $23(9.7 \%)$ & $0.017 *$ \\
\hline
\end{tabular}

eGFR estimated glomerular filtration rate, $h p f$ high powered field, RPGN rapidly progressive nephritic syndrome, HUS/TTP hemolytic uremic syndrome/thrombotic thrombocytopenic purpura, $M P O-A N C A$ myeloperoxidaseantineutrophil cytoplasmic antibody

MPGN commonly presents in childhood, but can be observed at all ages. Adult and elderly patients with MPGN had a higher frequency of nephrotic syndrome, clinical hypertension, heavy proteinuria, and hypoalbuminemia at the time of biopsy than that observed in children with MPGN.
The incidence of MPGN has been reported to decrease over time from about $7-10 \%$ in the 1970 s to about $2 \%$ during 1990-2011 [3-6, 16-19]. In the present study, among the 26,535 patients with biopsy-confirmed disease, 593 (2.2\%) patients were registered as having a histopathology of MPGN types I and III. Further, 332 out of the 26,535 


\section{a}

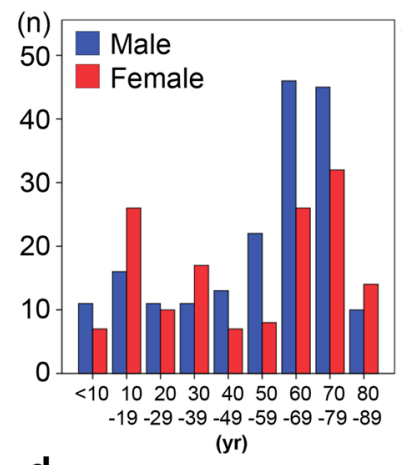

d

aged under 20 years

\begin{tabular}{|c|c|c|c|c|}
\hline Stage & A1 & A2 & A3 & Subtotal \\
\hline G1 & $\begin{array}{c}7 \\
(14.0 \%)\end{array}$ & $\begin{array}{c}4 \\
(8.0 \%)\end{array}$ & $\begin{array}{c}25 \\
(50.0 \%)\end{array}$ & $\begin{array}{c}36 \\
(72.0 \%)\end{array}$ \\
\hline \multirow{2}{*}{ G2 } & $\begin{array}{c}1 \\
(2.0 \%)\end{array}$ & $\begin{array}{c}1 \\
(2.0 \%)\end{array}$ & $\begin{array}{c}10 \\
(20.0 \%)\end{array}$ & $\begin{array}{c}12 \\
(24.0 \%)\end{array}$ \\
\hline \multirow{2}{*}{ G3a } & 0 & 0 & 0 & 0 \\
& $(0.0 \%)$ & $(0.0 \%)$ & $0.0 \%)$ & $(0.0 \%)$ \\
\hline G3b & 0 & 0 & 0 & 0 \\
& $(0.0 \%)$ & $(0.0 \%)$ & $(0.0 \%)$ & $(0.0 \%)$ \\
\hline \multirow{2}{*}{ G4 } & 1 & 0 & 0 & 1 \\
& $(2.0 \%)$ & $(0.0 \%)$ & $(0.0 \%)$ & $(2.0 \%)$ \\
\hline \multirow{2}{*}{ G5 } & 0 & 1 & 0 & 1 \\
& $(0.0 \%)$ & $(2.0 \%)$ & $(0.0 \%)$ & $(2.0 \%)$ \\
\hline Subtotal & 9 & 6 & 35 & $2 / 50$ \\
& $(18.0 \%)$ & $(12.0 \%)$ & $(70.0 \%)$ & $(4.0 \% / 100 \%)$ \\
\hline
\end{tabular}

\section{b}

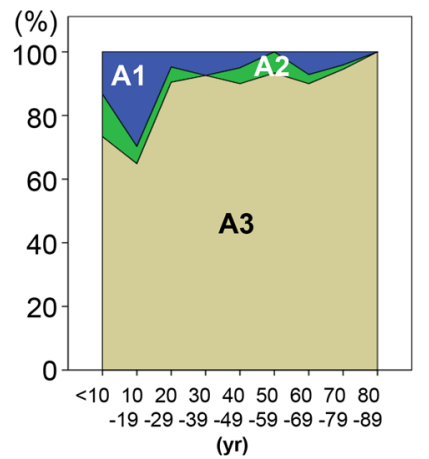

e

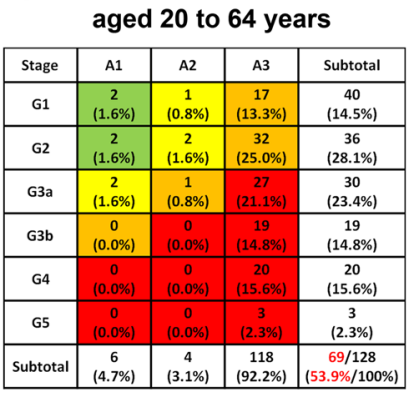

\section{c}

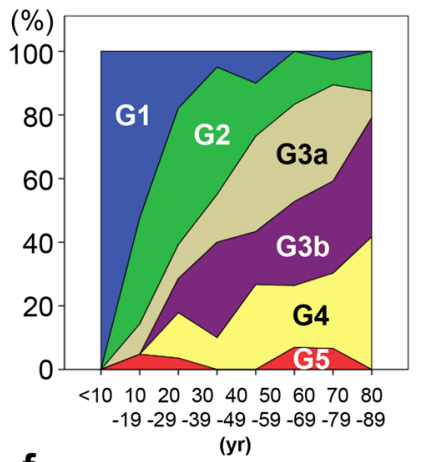

f

aged over 65 years

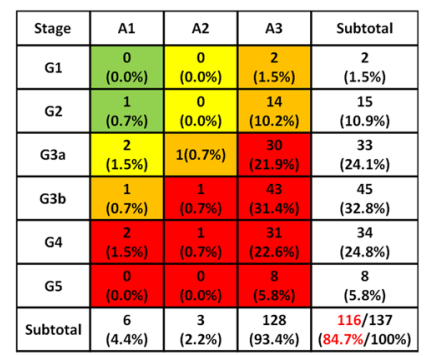

Fig. 2 Age distribution and clinical features of primary membranoproliferative glomerulonephritis (MPGN). a The number of cases peaked in the seventh decade for male patients, whereas they peaked in the eighth decade for the overall patients and female patients. b-c Chronic kidney disease (CKD) A stages (b) and G stages (c) of primary MPGN. More than $90 \%$ of patients aged more than 20 years had severe proteinuria (A3) (b). The number of patients with advanced

Table 5 Patient demographics of primary MPGN in Japan (J-RBR 2007-2015)

\begin{tabular}{llllll}
\hline & $N$ & Min & Max & Mean & SD \\
\hline Age (years old) & 332 & 3 & 89 & 51.6 & 24.6 \\
Male & 185 & 3 & 89 & 53.5 & 23.1 \\
Female & 147 & 4 & 87 & 49.2 & 26.4 \\
Height (cm) & 328 & 94.5 & 183.0 & 156.3 & 14.3 \\
Weight (kg) & 327 & 15.2 & 102.6 & 55.3 & 14.0 \\
Body mass index (BMI) & 327 & 10.4 & 34.9 & 22.3 & 3.8 \\
Proteinuria (g/day) & 232 & 0.0 & 19.4 & 3.8 & 3.4 \\
Urinary protein/creatinine ratio & 250 & 0.0 & 32.0 & 5.0 & 4.6 \\
Serum creatinine (mg/dl) & 332 & 0.2 & 9.7 & 1.3 & 1.1 \\
eGFR (ml/min/1.73 m $\left.{ }^{2}\right)$ & 328 & 6.4 & 161.3 & 59.0 & 33.8 \\
Serum total protein (g/dl) & 332 & 3.3 & 8.7 & 5.7 & 1.0 \\
Serum albumin (g/dl) & 331 & 1.1 & 5.0 & 3.0 & 0.8 \\
Serum total cholesterol (mg/dl) & 324 & 78.0 & 558.0 & 230.7 & 73.3 \\
Systolic blood pressure (mmHg) & 284 & 87.0 & 197.0 & 137.9 & 22.4 \\
Diastolic blood pressure (mmHg) & 284 & 42.0 & 114.0 & 76.1 & 13.4 \\
Mean blood pressure (mmHg) & 284 & 57.0 & 135.0 & 96.7 & 14.5 \\
HbAlc (\%) & 192 & 3.8 & 7.4 & 5.6 & 0.5 \\
\hline
\end{tabular}

$J-R B R$ Japan Renal Biopsy Registry, eGFR estimated glomerular filtration rate, $H b A l c$ hemoglobin A1c
CKD (stage G3a, G3b, G4, or G5) increased with age, and more than $70 \%$ of them were 60 years old or older $(\mathbf{c})$. d-f The CGA risk classification of primary MPGN. According to the CGA risk classification, high-risk (red zone) cases accounted for $4.0 \%$ of patients aged under 20 years (d), $53.9 \%$ of patients aged 20 to 64 years (e) and $84.7 \%$ of those aged over 65 years (f)

(1.3\%) patients were registered as primary MPGN. In a comprehensive epidemiological study, the lesion of MPGN was observed in about $2 \%$ of patients with biopsy-confirmed glomerulonephritis in the Australia [3] and United States [19]. As for primary MPGN, a recent report from Japan has showed a $1.2 \%$ incidence of primary MPGN in a total of 6,369 renal biopsies [17]. The present study confirmed these findings.

MPGN is the sixth leading cause of end-stage renal disease among the glomerulonephritides and the fifth leading cause of end-stage renal disease among the primary glomerular diseases, as reported in an international comparative study including patients in the United States, Europe, and Australia/New Zealand [16]. In the present study, according to the CGA risk classification, high-risk (red zone) cases accounted only for $3.4 \%$ of children with MPGN, whereas these cases accounted for 52.5 and $84.1 \%$ of adult and elderly patients with overall MPGN, respectively. Similarly, these cases accounted for $4.0 \%$ of children, $53.9 \%$ of adults and $84.7 \%$ of elderly patients with primary MPGN. These results suggest that MPGN is an important disorder leading to end-stage renal disease, even in Japan. Further, higher value for systolic BP and serum total cholesterol, and 
Table 6 Comparison of clinical features and diagnosis among age group in primary MPGN

\begin{tabular}{lllll}
\hline & Child & Adult & Elderly & $P$ \\
\hline$N$ & 60 & 129 & 143 & \\
Age (years old) & $11.4 \pm 4.2$ & $45.8 \pm 13.7$ & $73.6 \pm 5.6$ & $<0.001^{*}$ \\
Male/Female & $27 / 33$ & $79 / 50$ & $79 / 64$ & 0.111 \\
Body mass index (BMI) & $18.9 \pm 3.3$ & $22.8 \pm 3.2$ & $23.3 \pm 3.7$ & $<0.001^{*}$ \\
Systolic blood pressure (mmHg) & $112.8 \pm 15.8$ & $136.0 \pm 18.1$ & $148.2 \pm 20.3$ & $<0.001^{*}$ \\
Diastolic blood pressure (mmHg) & $64.4 \pm 11.5$ & $79.3 \pm 12.5$ & $77.3 \pm 12.8$ & $<0.001^{*}$ \\
Patients with hypertension at diagnosis & $13.3 \%$ & $51.2 \%$ & $67.8 \%$ & $<0.001^{*}$ \\
Sediment RBC (>30/hpf, \%) & $38.3 \%$ & $23.3 \%$ & $32.2 \%$ & 0.078 \\
Proteinuria (g/day) & $1.8 \pm 2.2$ & $4.0 \pm 3.2$ & $4.2 \pm 3.6$ & $0.003^{*}$ \\
Urinary protein/creatinine ratio & $2.6 \pm 4.2$ & $4.5 \pm 3.4$ & $6.2 \pm 5.2$ & $<0.001^{*}$ \\
Serum creatinine (mg/dl) & $0.75 \pm 1.23$ & $1.29 \pm 0.85$ & $1.56 \pm 1.06$ & $<0.001^{*}$ \\
eGFR (ml/min/1.73 m $\left.{ }^{2}\right)$ & $104.2 \pm 30.5$ & $59.6 \pm 28.7$ & $40.2 \pm 18.6$ & $<0.001^{*}$ \\
Serum albumin (g/dl) & $3.5 \pm 0.9$ & $3.0 \pm 0.8$ & $2.8 \pm 0.7$ & $<0.001^{*}$ \\
Serum total cholesterol (mg/dl) & $219.1 \pm 73.4$ & $237.9 \pm 76.1$ & $229.3 \pm 70.3$ & 0.251 \\
Clinical diagnosis & & & & \\
Nephrotic syndrome & $10(16.7 \%)$ & $63(48.8 \%)$ & $90(62.9 \%)$ & $<0.001^{*}$ \\
Chronic nephritic syndrome & $46(76.7 \%)$ & $60(46.5 \%)$ & $42(29.4 \%)$ & $<0.001^{*}$ \\
Rapidly progressive nephritic syndrome & $1(1.7 \%)$ & $3(2.3 \%)$ & $6(4.2 \%)$ & 0.531 \\
Acute nephritic syndrome & $0(0.0 \%)$ & $1(0.8 \%)$ & $1(0.7 \%)$ & 0.798 \\
Recurrent hematuria & $2(3.3 \%)$ & $1(0.8 \%)$ & $0(0.0 \%)$ & 0.163 \\
Acute kidney injury & $0(0.0 \%)$ & $1(0.8 \%)$ & $1(0.7 \%)$ & 0.798 \\
\hline
\end{tabular}

$e G F R$ estimated glomerular filtration rate, $h p f$ high powered field
Table 7 Impact of clinical features and pathogenesis on renal function at the time of biopsy in adult and elderly patients with membranoproliferative glomerulonephritis (MPGN)

\begin{tabular}{llll}
\hline Parameters at diagnosis & Standard b & $\mathrm{t}$ & $P$ \\
\hline (a) MPGN & & & \\
$\quad$ Systolic blood pressure & -0.248 & -4.740 & $<0.001$ \\
Proteinuria (g/day) & -0.177 & -3.255 & 0.001 \\
$\quad$ Serum total cholesterol & 0.108 & 1.998 & 0.046 \\
$R^{2}=0.104 ; F=13.021, p<0.001 *$ & & & \\
(b) Primary MPGN & & & \\
Systolic blood pressure & -0.299 & -4.254 & $<0.001$ \\
Proteinuria (g/day) & -0.216 & -2.957 & 0.004 \\
Serum total cholesterol & 0.147 & 2.030 & 0.044 \\
$R^{2}=0.157 ; F=10.791, p<0.001 *$ & & & \\
\hline
\end{tabular}

*Statistically significant by multiple regression analysis (stepwise method)

increased proteinuria at diagnosis were significant factors for a decline in renal function in adult and elderly patients not only with overall MPGN but also with primary MPGN, suggesting that management of BP might be important for retarding the decline of renal function in patients with MPGN.

The reason why the older patients had more proteinuria and worse renal function might be that the older patients had MPGN for a long time or late stage, although we have no data about the duration between disease onset and renal biopsy. Moreover, the present data including glomerulonephritides with established disease entities such as lupus nephritis, IgA nephropathy, and MPO-ANCA-positive nephritis might be difficult to interpret legitimately to evaluate the clinical features of MPGN, because these diseases generally show different clinical features and develop in different age groups.

Primary MPGN is a diagnosis of exclusion, at least in many adults and some children, and a systematic approach to evaluation will often uncover a secondary cause, such as an infection, autoimmune disease, monoclonal gammopathy, neoplasia, complement dysregulation, or a chronic thrombotic microangiopathy $[1,9]$. Several studies have indicated that secondary MPGN is most often due to hepatitis $\mathrm{C}$ and other infections $[1,9]$. Although the prevalence and age distribution of secondary MPGN had remained uncertain, we clearly showed that lupus nephritis was the most common disease among patients with secondary MPGN patients aged 20-39 years, whereas infectious disease was the most common among patients aged 40 years and above.

This study has some limitations. First, we cannot exclude the possibility that the J-RBR is subject to sampling bias; however, the registry contributes to not only the standardization of histological diagnosis and classification, but also to nationwide epidemiological studies of conditions such as nephrotic syndrome and glomerulonephritis [6, 
20-22]. Thus, it is likely to be reasonably representative of the nationwide situation of renal biopsied cases in Japan. Second, the registry data might be somewhat inaccurate. Although it is necessary to exclude the secondary cause of MPGN before confirming a diagnosis of primary MPGN, we cannot exclude the possibility that some patients who were registered as primary MPGN had underlying infectious diseases, autoimmune diseases, or paraproteins at the time of registration. Third, the number of items on the registration form was so definitive that we were unable to evaluate the immunological data; for example, complements, antinuclear antibody, and immunofluorescence findings of the renal biopsies, although the diagnosis of MPGN has recently changed from an electron microscopy-based ultrastructural classification scheme to one based largely on immunofluorescence findings [1, 23, 24]. Finally, this cross-sectional study had no reference to the duration between disease onset and renal biopsy, the treatments and outcomes of any of the patients. However, the baseline data of this study have a potential to be helpful in further studies such as a longitudinal cohort study. From this perspective, a large longitudinal cohort study should be planned to clarify and compare the actual outcomes in MPGN and C3 glomerulopathy.

In conclusion, the frequency of patients who were registered as MPGN peaked between the ages of 60 and 79 years, and adults and elderly patients with MPGN had a lower eGFR and severer proteinuria than children, in the Japanese nationwide registry. The clinical characteristics and actual renal outcomes of patients with MPGN and C3 glomerulopathy require further longitudinal investigation.

Acknowledgements The authors are grateful to all their colleagues who participated in the J-RBR (Supplementary Appendix). This study was supported in part by the committee of the Japanese Society of Nephrology and in part by a Grant-in-Aid for Intractable Renal Diseases Research, Research on rare and intractable diseases, Health and Labour Sciences Research Grants from the Ministry of Health, Labour, and Welfare of Japan.

Ethical standards The ethical committee of the Japanese Society of Nephrology comprehensively examined and approved the study protocol. The J-RBR is registered at the Clinical Trial Registry of UMIN (UMIN000000618).

Conflict of interest The authors have no conflicts of interest to declare.

Informed consent Informed consent was obtained from all individual participants included in the study.

Open Access This article is distributed under the terms of the Creative Commons Attribution 4.0 International License (http://creativecommons.org/licenses/by/4.0/), which permits unrestricted use, distribution, and reproduction in any medium, provided you give appropriate credit to the original author(s) and the source, provide a link to the Creative Commons license, and indicate if changes were made.

\section{References}

1. ADDIN EN.REFLIST 1. Sethi S, Fervenza FC. Membranoproliferative glomerulonephritis - a new look at an old entity. N Engl J Med. 2012;366(12):1119-31.

2. Masani N, Jhaveri KD, Fishbane S. Update on membranoproliferative GN. Clin J Am Soc Nephrol. 2014;9(3):600-8.

3. Briganti EM, Dowling J, Finlay M, Hill PA, Jones CL, KincaidSmith PS, et al. The incidence of biopsy-proven glomerulonephritis in Australia. Nephrol Dial Transplant. 2001;16(7):1364-7.

4. Swaminathan S, Leung N, Lager DJ, Melton LJ 3rd, Bergstralh EJ, Rohlinger A, et al. Changing incidence of glomerular disease in Olmsted County, Minnesota: a 30-year renal biopsy study. Clin J Am Soc Nephrol. 2006;1(3):483-7.

5. Zhou FD, Zhao MH, Zou WZ, Liu G, Wang H. The changing spectrum of primary glomerular diseases within 15 years: a survey of 3331 patients in a single Chinese centre. Nephrol Dial Transplant. 2009;24(3):870-6.

6. Yokoyama H, Taguchi T, Sugiyama H, Sato H. Membranous nephropathy in Japan: analysis of the Japan Renal Biopsy Registry (J-RBR). Clin Exp Nephrol. 2012;16(4):557-63.

7. Sethi S, Nester CM, Smith RJ. Membranoproliferative glomerulonephritis and $\mathrm{C} 3$ glomerulopathy: resolving the confusion. Kidney Int. 2012;81(5):434-41.

8. Ito N, Ohashi R, Nagata M. C3 glomerulopathy and current dilemmas. Clin Exp Nephrol. 2017;21(4):541-51.

9. Fervenza FC, Sethi S, Glassock RJ. Idiopathic membranoproliferative glomerulonephritis: does it exist? Nephrol Dial Transplant. 2012;27(12):4288-94.

10. Sugiyama H, Yokoyama H, Sato H, Saito T, Kohda Y, Nishi S, et al. Japan Renal Biopsy Registry: the first nationwide, webbased, and prospective registry system of renal biopsies in Japan. Clin Exp Nephrol. 2011;15(4):493-503.

11. Uemura $\mathrm{O}$, Nagai $\mathrm{T}$, Ishikura $\mathrm{K}$, Ito $\mathrm{S}$, Hataya $\mathrm{H}$, Gotoh $\mathrm{Y}$, et al. Creatinine-based equation to estimate the glomerular filtration rate in Japanese children and adolescents with chronic kidney disease. Clin Exp Nephrol. 2014;18(4):626-33.

12. Matsuo S, Imai E, Horio M, Yasuda Y, Tomita K, Nitta K, et al. Revised equations for estimated GFR from serum creatinine in Japan. Am J Kidney Dis. 2009;53(6):982-92.

13. Yokoyama H, Narita I, Sugiyama H, Nagata M, Sato H, Ueda Y, et al. Drug-induced kidney disease: a study of the Japan Renal Biopsy Registry from 2007 to 2015. Clin Exp Nephrol. 2015;20(5):720-30.

14. Nishi S, Ubara Y, Utsunomiya Y, Okada K, Obata Y, Kai H, et al. Evidence-based clinical practice guidelines for nephrotic syndrome 2014. Clin Exp Nephrol. 2016;20(3):342-70.

15. Cameron JS, Turner DR, Heaton J, Williams DG, Ogg CS, Chantler C, et al. Idiopathic mesangiocapillary glomerulonephritis. Comparison of types I and II in children and adults and longterm prognosis. Am J Med. 1983;74(2):175-92.

16. Maisonneuve P, Agodoa L, Gellert R, Stewart JH, Buccianti G, Lowenfels $\mathrm{AB}$, et al. Distribution of primary renal diseases leading to end-stage renal failure in the United States, Europe, and Australia/New Zealand: results from an international comparative study. Am J Kidney Dis. 2000;35(1):157-65.

17. Kawamura T, Usui J, Kaseda K, Takada K, Ebihara I, Ishizu T, et al. Primary membranoproliferative glomerulonephritis on the decline: decreased rate from the 1970s to the 2000s in Japan. Clin Exp Nephrol. 2013;17(2):248-54.

18. McGrogan A, Franssen CF, de Vries CS. The incidence of primary glomerulonephritis worldwide: a systematic review of the literature. Nephrol Dial Transplant. 2011;26(2):414-30.

19. Sim JJ, Batech M, Hever A, Harrison TN, Avelar T, Kanter MH, et al. Distribution of Biopsy-Proven Presumed 
Primary Glomerulonephropathies in 2000-2011 among a racially and ethnically diverse US population. Am J Kidney Dis. 2016;68(4):533-44.

20. Komatsu H, Fujimoto S, Yoshikawa N, Kitamura H, Sugiyama $\mathrm{H}$, Yokoyama H. Clinical manifestations of Henoch-Schonlein purpura nephritis and IgA nephropathy: comparative analysis of data from the Japan Renal Biopsy Registry (J-RBR). Clin Exp Nephrol. 2016;20(4):552-60.

21. Hiromura K, Ikeuchi H, Kayakabe K, Sugiyama H, Nagata M, Sato $\mathrm{H}$, et al. Clinical and histological features of lupus nephritis in Japan: a cross-sectional analysis of the Japan Renal Biopsy Registry (J-RBR). Nephrology (Carlton). 2016.
22. Nishi S, Muso E, Shimizu A, Sugiyama H, Yokoyama H, Ando $\mathrm{Y}$, et al. A clinical evaluation of renal amyloidosis in the Japan renal biopsy registry: a cross-sectional study. Clin Exp Nephrol. 2017;21(4):624-32.

23. Larsen CP, Messias NC, Walker PD, Fidler ME, Cornell LD, Hernandez LH, et al. Membranoproliferative glomerulonephritis with masked monotypic immunoglobulin deposits. Kidney Int. 2015;88(4):867-73.

24. Sethi S, Haas M, Markowitz GS, D'Agati VD, Rennke HG, Jennette JC, et al. Mayo Clinic/Renal Pathology Society Consensus Report on Pathologic Classification, Diagnosis, and Reporting of GN. J Am Soc Nephrol. 2016;27(5):1278-87. 